\title{
Aporte tecnológico al desarrollo de la quesera principal de Salinas, en la provincia de Bolívar
}

\author{
Technological Contribution to the Growth of the Main Cheese \\ Factory in Salinas, Province of Bolivar
}

Aporte tecnológico ao desenvolvimento da queijeira principal de Salinas, província de Bolívar

\author{
Adriana Delgado \\ Instituto de Altos Estudios Nacionales. Quito, Ecuador \\ adriana_delgadog@hotmail.com \\ https://orcid.org/0000-0002-1271-6802
}

DOI: https://doi.org/10.32719/25506641.2022.11.4

Recibido: 15 de enero de 2021 - Revisado: 12 de marzo de 2021

Aceptado: 22 de abril de 2021 • Publicado: 1 de enero de 2022

Artículo de investigación 


\section{Adriana Delgado}

\section{Resumen}

Este artículo presenta los resultados de una investigación que tuvo como objetivo establecer el aporte de la tecnología en el desarrollo de la quesera principal de Salinas en la provincia de Bolívar, mediante la contribución y la relevancia de su uso. En este contexto, contempla una primera aproximación a la trasferencia de conocimiento y tecnología realizada por José Dubach en el proceso de elaboración de quesos. El alcance de la investigación fue de tipo exploratorio-descriptivo e involucra un análisis mixto (cualitativo y cuantitativo) para dar cuenta del impacto positivo que tuvo para las comunidades la implementación tecnológica y el posterior desarrollo de El Salinerito. El resultado ofrece una visión general y amplia de la aplicación de tecnologías humanas, mecánicas y del conocimiento para el desarrollo y posterior progreso de las comunidades, permitiendo superar las condiciones de extrema pobreza por ingresos de los campesinos salineros y lograr un desarrollo social sostenible en casi cuarenta años desde su creación hasta la actualidad. Con este análisis se abre la posibilidad de visibilizar este tipo de procesos como casos exitosos que permiten mejorar la calidad de vida de las comunidades, y suponen la base para el desarrollo de actividades y proyectos similares en otras zonas.

Palabras clave: Tecnología, transferencia de conocimiento y tecnología, desarrollo social, impacto social, El Salinerito.

JEL: O31 Innovación e invención: procesos e incentivos.

\section{Abstract}

This article presents the results of a study, whose main purpose was to determine the contribution of technology in the growth of the main Cheese Factory in Salinas, Province of Bolívar, based on the contribution and relevance in the use thereof. In this context, the study includes a first glance by José Dubach at the transfer of knowledge and technology in the cheese-making process. The scope of research was exploratory-descriptive in nature, and it involves a mixed analysis (qualitative and quantitative) to account for the positive impact on the communities thanks to the technological implementation and the subsequent development of El Salinerito. The result offers a broad overview of the application of human, mechanical, and knowledge technologies for the development and subsequent progress of the communities, allowing them to overcome the conditions of extreme poverty thanks to the income of the salt farmers, and thereby achieving sustainable social development in almost forty years from its inception to this date. This analysis creates more visibility for these types of processes as successful cases that improve the quality of life of the communities, which represent the basis for carrying out similar activities and projects in other areas.

Keywords: Technology, transfer of knowledge and technology, social development, social impact, El Salinerito.

JEL: O31 Innovation and invention: processes and incentives. 


\section{Resumo}

Este artigo apresenta os resultados de uma investigação que teve como objetivo principal estabelecer a contribuição da tecnologia no desenvolvimento da Queijeira principal de Salinas da província de Bolívar, mediante o aporte e a relevância de seu uso. Neste contexto, contempla uma primeira aproximação à transferência de conhecimento e tecnologia realizada por José Dubach no processo de elaboração de queijos. O alcance da investigação foi do tipo exploratória-descritiva e envolve uma análise mista (qualitativa e quantitativa) para dar conta do impacto positivo que teve para as comunidades a implementação tecnológica e o posterior desenvolvimento de El Salinerito. O resultado oferece uma visão geral e ampla da aplicação de tecnologias humanas, tecnologias mecânicas e tecnologias do conhecimento para o desenvolvimento e posterior progresso das comunidades, permitindo superar as condições de extrema pobreza pelos ingressos dos camponeses salineiros e obter um desenvolvimento social sustentável em quase quarenta anos desde sua criação até a atualidade. Com esta análise se abre a possibilidade de visibilizar este tipo de processos como casos exitosos que permitem melhorar a qualidade de vida das comunidades, e supõem a base para o desenvolvimento de atividades e projetos similares noutras zonas.

Palavras-chave: Tecnologia, transferência de conhecimento e tecnologia, desenvolvimento social, impacto social, El Salinerito.

JEL: O31 Inovação e invenção: processos e incentivos.

\section{Introducción}

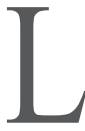

a parroquia de Salinas de Tomabela está ubicada en el centro de la Sierra ecuatoriana en la provincia de Bolívar del cantón Guaranda, con una población total de 7262 habitantes en 2014 (EC SENPLADES 2015). El nombre Salinas se origina por la presencia de minas de sal cerca de la cabecera parroquial. Desde la época precolombina hasta la década de 1970, la principal actividad económica de su población era recabar la sal de las vertientes de agua salada para procesarla y obtenerla en estado sólido, que posteriormente era comercializada mediante el trueque a cambio de diversos productos no disponibles en la zona, como legumbres, frutas, panela entre otros (Polo 2002). Esta actividad de procesamiento y comercialización se realizaba bajo el mandato de los latifundistas de la familia Cordobés, quienes recibían tributo debido a que las minas de sal se localizaban en sus haciendas. Sin embargo, la Reforma Agraria de la década de 1970, que estableció la nacionalización de los recursos naturales, desencadenó la migración de la 
población a otras actividades económicas debido a la fuerte competencia que representaron las grandes empresas productoras de sal en la región.

En 1971, por pedido del monseñor Cándido Rada, obispo de Guaranda, llegó la Operación Mato Grosso (OMG) con una misión Salesiana encabezada por el padre Antonio Polo, quien encontró en Salinas una población sumida en la extrema pobreza, carente de diversos servicios básicos, como servicios públicos, educación, salud, entre otros. En ese momento, se inició un nuevo proceso de desarrollo comunitario en Salinas, aprovechando las iniciativas locales y los niveles de organización comunitaria para el desarrollo de proyectos productivos. Las primeras actividades en 1972 llevaron a la formación de la Cooperativa Salinas, que posteriormente se convertiría en una Cooperativa de Ahorro y Crédito cuyo fin era apoyar las actividades productivas de sus socios (Naranjo 2012). En 1977, se creó el grupo juvenil y en 1982, la unión de Organizaciones de Salinas (UNORSAL), convertidas sucesivamente en fundaciones (Fundación Juvenil y FUNORSAL).

En ese contexto, el padre Antonio Polo, decidido a contribuir con la disminución de los niveles de pobreza de los habitantes de la zona a través del desarrollo de una infraestructura mínima (Polo 2002), impulsó la creación de la primera quesera en la cabecera parroquial, establecida en 1978. Esta se inauguró como resultado de un acuerdo bilateral celebrado entre los Gobiernos de Ecuador y de Suiza, contando con la asesoría principal del suizo José Dubach, financiada por una donación de la Cooperación Técnica Suiza (COTESU), créditos del Fondo Ecuatoriano Populorium Progresio (FEPP) y apoyada por la Promoción Humana de la Diócesis de Guaranda.

La primera quesera institucionalizada, El Salinerito, marcó un precedente en el progreso de la comunidad salinera, pues la incorporación de transferencia de técnicas y tecnologías modernas (Sagasti 2011) y el desarrollo de valor agregado a los recursos obtenidos, le permitió evolucionar hasta convertirse en un referente de la producción de lácteos en Ecuador. De esta forma, logró crear un fuerte mercado competitivo basado en un modelo de economía solidaria que buscaba el mejoramiento de los ingresos y el incremento del nivel del empleo, educación, salud y vivienda de la comunidad (North 2008).

Esto se logró a través de la transferencia de conocimiento liderada por José Dubach, que cortó con la racionalidad del mercado capitalista, impar- 
tiendo un avance en la infraestructura tecnológica, dotando a la población salinera de un conjunto de respuestas organizadas para afrontar su entorno biofísico y social (Sagasti 2011). Estos factores dieron lugar a la transformación y expansión de su actividad productiva con lo que se pudo desarrollar un queso de calidad bajo la marca de El Salinerito (Naranjo 2012).

El progreso salinero se puede asociar al desarrollo social, como plantea Escobar (2014), como un elemento fundamental de las necesidades y aspiraciones de las personas para aprovechar capacidades, recursos y oportunidades con el fin de obtener nuevos conocimientos que permitan el acceso a recursos que provean un nivel de vida aceptable. La investigación realizada tuvo como punto de partida esta idea para el estudio de los aportes de la tecnología en el desarrollo de la quesera principal de Salinas de la provincia de Bolívar, contemplando una primera aproximación a la trasferencia de conocimiento y tecnología por José Dubach en el proceso de elaboración de quesos.

\section{Marco teórico}

\section{Transferencia de conocimiento}

Esta investigación se fundamenta en la trasferencia del conocimiento entendida como "el movimiento y difusión de una tecnología o producto desde su invención original a un contexto económico y social diferente" (Becerra 2004, 70).

El conocimiento es considerado como un objeto, "el cual puede ser observado, almacenado, usado y reutilizado, o como un proceso o flujo de interacciones que involucran aspectos cognitivos y del aprendizaje" (Albino, Garavelli y Gorgoglione 2004, 34). El conocimiento científico y tecnológico, tácito o explícito, "constituyen las salidas del proceso de desarrollo, el cual toma la forma de publicaciones, procesos, materiales, tecnología, know-how, innovación y habilidades" (Libraryhouse 2008, 46).

El proceso de transferencia está compuesto por una serie de actividades, mecanismos o canales, mediante los cuales este llega al Estado, a la industria 
o a las comunidades generando un impacto económico, social y un mejoramiento del conocimiento mismo.

\section{Tecnología}

La tecnología es uno de los desarrollos más avanzados que ha tenido la sociedad actualmente y gracias a la cual se ha desplegado una gran cantidad de posibilidades, convirtiéndose en un pilar fundamental en la vida de muchos individuos, dado que los avances tecnológicos han supuesto soluciones y respuestas óptimas a diversas necesidades.

La tecnología "es un tipo de conocimiento en el cual el saber-cómo define el grado de efectividad que se pueda alcanzar en un ámbito específico" (Jarvie 2004, 57). Abarca dentro de sí el proceso de hacer algo que es realizable (invención), implementación de la ciencia aplicada, así como invención y mantenimiento de artefactos mecánicos.

La tecnología está constituida por las herramientas desarrolladas por el inventor y representa un saber-cómo más que un saber-qué, intentando explorar y explicar los detalles del entorno, por lo cual también es específica del entorno. Según Price (1980), la tecnología es aquella investigación cuyo producto principal es una máquina, un medicamento, un producto o un proceso de algún tipo.

En la sociedad contemporánea, las máquinas de cambio más poderosas son la invención humana, la innovación y las aplicaciones del conocimiento científico. Colectivamente, se denomina a estas funciones como de tecnología (Wenk 1989). Integran las visiones en los sistemas complejos y "proporcionan un paradigma integral de la tecnología, sugiriendo que tales sistemas son el conjunto de tecnologías mecánicas, de conocimiento y humanas usadas para transformar inputs en outputs en el sector productivo" (Collins, Hage y Hull 1988, 87).

De acuerdo con el primer significado etimológico, la tecnología tiene que ser la teoría, la ciencia, el estudio, la discusión de la técnica, cubiertas en esta última, las habilidades del hacer, las profesiones y, generosamente, los modos de producir algo. Este es necesariamente el sentido primordial, cuya interpretación nos abrirá la comprensión de los demás. La tecnología aparece aquí como el valor fundamental y exacto de logotipos de la técnica (Vieira 
Pinto 2008). En este orden de ideas, la tecnología se puede clasificar como: mecánica, humana y del conocimiento (Collins, Hage y Hull 1988).

Las tecnologías mecánicas se refieren a las máquinas, herramientas y equipos usados para producir bienes que caracterizan la propia tecnología en la mayoría de los enfoques (Roberts y Grabowsky 1996). Estas tecnologías consideran la aplicación de la mecánica física, por tanto, se ocupa del estudio de las fuerzas y movimientos de los sistemas mecánicos y su construcción, lo que se traduce en la construcción de herramientas o artefactos (González García, Luján y López Cerezo 1996).

En esta tecnología se estudian "los procesos de conformado y fabricación de componentes mecánicos con la adecuada precisión dimensional, así como de la maquinaria, herramientas y demás equipos necesarios para llevar a cabo la realización física de tales procesos, su automatización, planificación y verificación" (Departamento de Ingeniería Marítima de España 2018).

Las tecnologías humanas consisten en las habilidades y la energía física usadas en la producción de bienes y pueden ser sustituidas por tecnologías mecánicas (Roberts y Grabowsky 1996).

Bajo la clasificación de tecnologías del conocimiento, se comprenden los significados y los conceptos abstractos usados en la producción y cómo las tecnologías del conocimiento pueden restringir o facilitar el desenvolvimiento y la utilización de tecnologías mecánicas y humanas, considerándolas las más importantes (Perrow 1967). Es interesante observar que cada una de estas tecnologías es interdependiente: coexisten e interrelacionan en sistemas y organizaciones que ejercen un impacto significativo sobre las prácticas actuales y futuras (Roberts y Grabowsky 1996).

La implementación de la tecnología ayuda a contrarrestar los problemas prácticos establecidos por la sociedad en diversos ámbitos del entorno, medioambiente y espacio. Así las cosas, la trasferencia de tecnología se fundamentará en la trasferencia de esa capacidad tecnológica para adaptar y mejorar la tecnología existente.

La transferencia de tecnología es "dotar al individuo que ya tienen esta infraestructura básica, de los repertorios conductuales suficientes para tener éxito en su trabajo, desde la operación hasta el rediseño, asociándolo como un aprendizaje de un arte, dominio de un instrumento o un proceso escolarizado" (Olarte 1996, 38). Por otro lado, la transferencia "hace referencia a un 
proceso mediante el cual la ciencia y la tecnología se difunden en las actividades humanas y el proceso de incorporación a una unidad productiva de un conocimiento desarrollado fuera de ella" (Tapias-García 2016, 9).

\section{Metodología}

La investigación busca determinar cómo la trasferencia de conocimiento por José Dubach en la industria quesera, utilizando tecnologías en las etapas del proceso comunitario de Salinas de Guaranda, surtieron un impacto social en las comunidades.

La población de Salinas de Guaranda de la provincia de Bolívar, cuenta con 25 comunidades con presencia de una quesera rural. Fueron seleccionadas dos unidades de análisis: la primera, la quesera principal en la cabecera parroquial, El Salinerito, y la segunda, una muestra estratificada guiada por propósitos de las 25 comunidades de la parroquia de salinas incluyendo el cantón parroquial, que contaban con queseras rurales.

Se consideró un muestreo mixto estratificado por propósitos. Una muestra estratificada guiada por propósitos implica segmentar la población de intereses en estratos (que constituyen una acción probabilística) y luego seleccionar en cada subgrupo un número relativamente pequeño de casos para estudiarlos (Teddlie y Yu 2007). Yamane (1967) provee una formula simplificada para determinar el tamaño de la muestra, donde $n$ es el tamaño de la muestra, $N$ es el tamaño de la población y $e$ es el tamaño del error permitido para este caso del $95 \%, \mathrm{e}=0,05$.

$$
n=\frac{N}{1+\mathrm{Ne}^{2}}
$$

Aplicado a la población de Salinas de Guaranda, según el equipo técnico del GAD Salinas (2015), se tiene una población de 6919 personas. Para determinar la muestra se emplearon los siguientes datos: Población $(\mathrm{N})=6919$; Error admisible $=0,05$; lo que da como resultado una muestra de 378 . Sin embargo, considerando las condiciones de muestreo se eligió una muestra estratificada proporcional de 195. La distribución de la muestra se evidencia en la tabla 1. 


\section{Tabla 1}

\section{Distribución de la muestra de las comunidades con queseras en Salinas de Guaranda}

\begin{tabular}{|c|c|c|c|}
\hline Población & Frecuencia & $\%$ & $\begin{array}{c}\% \\
\text { acumulado }\end{array}$ \\
\hline Apahua, población hasta 500 habitantes & 9 & 4,6 & 4,6 \\
\hline Calvario, población menor de 50 habitantes & 1 & 0,5 & 5,1 \\
\hline Cañitas, población menor de 50 habitantes & 3 & 1,5 & 6,7 \\
\hline Chaupi, población hasta con 100 habitantes & 2 & 1,0 & 7,7 \\
\hline Chazojuan, población hasta 500 habitantes & 13 & 6,7 & 14,4 \\
\hline Copalpamba, población hasta con 100 habitantes & 2 & 1,0 & 15,4 \\
\hline Gramalote, población hasta con 100 habitantes & 1 & 0,5 & 15,9 \\
\hline Guarumal, población menor de 50 habitantes & 1 & 0,5 & 16,4 \\
\hline La Libertad, población hasta 200 habitantes & 5 & 2,6 & 19,0 \\
\hline La Moya, población hasta 500 habitantes & 8 & 4,1 & 23,1 \\
\hline La Palma, población hasta 500 habitantes & 10 & 5,1 & 28,2 \\
\hline Pambabuela, población hasta 1500 habitantes & 22 & 11,3 & 39,5 \\
\hline Lanzaurco, población hasta 500 habitantes & 6 & 3,1 & 42,6 \\
\hline Arrayanes, población menor de 50 habitantes & 1 & 0,5 & 43,1 \\
\hline Matiaví Bajo, población hasta 200 habitantes & 4 & 2,1 & 45,1 \\
\hline Las Mercedes de Pumin, población hasta con 100 habitantes & 2 & 1,0 & 46,2 \\
\hline Rincón de los Andes, población hasta con 100 habitantes & 1 & 0,5 & 46,7 \\
\hline San Vicente de Plancha, población hasta con 100 habitantes & 2 & 1,0 & 47,7 \\
\hline Natawa, población hasta 200 habitantes & 4 & 2,1 & 49,7 \\
\hline Pachancho, población hasta 200 habitantes & 6 & 3,1 & 52,8 \\
\hline Verdepamba, población hasta 200 habitantes & 5 & 2,6 & 55,4 \\
\hline Monoloma, población hasta 500 habitantes & 7 & 3,6 & 59,0 \\
\hline Yuraucsha, población hasta 200 habitantes & 6 & 3,1 & 62,1 \\
\hline Piscoquero, población hasta 200 habitantes & 6 & 3,1 & 65,1 \\
\hline Mulidiahuán, población hasta 500 habitantes & 7 & 3,6 & 68,7 \\
\hline Tigreurco, población hasta 200 habitantes & 8 & 4,1 & 72,8 \\
\hline
\end{tabular}




\begin{tabular}{|l|r|r|r|}
\hline Yacubiana, población hasta 500 habitantes & 10 & 5,1 & 77,9 \\
\hline B. San Francisco, población hasta con 100 habitantes & 2 & 1,0 & 79,0 \\
\hline Nueva Esperanza, población hasta con 100 habitantes & 3 & 1,5 & 80,5 \\
\hline B. Estadio, población hasta 500 habitantes & 9 & 4,6 & 85,1 \\
\hline Centro Poblado, población hasta 1500 habitantes & 29 & 14,9 & 100,0 \\
\hline Total & 195 & 100,0 & \\
\hline
\end{tabular}

Elaboración propia.

Para determinar las medidas de coherecia o consitencia interna, se utilizó el coeficiente de confiabilidad Alfa de Cronbach. Este es un valor "entre 0,70 y 0,90 [que] indica una buena consistencia interna para una escala unidimensional" (Celina Oviedo y Campo Arias 2005, 574). En la tabla 2, se relacionan los coeficientes de Alfa de Cronbach respecto al cuestianario utilizado, se verifica la consitencia interna del formulario.

Tabla 2

Valores del Alfa de Cronbrach para validar el formulario

\begin{tabular}{|l|c|c|}
\hline \multicolumn{1}{|c|}{ Subcategoría } & $\begin{array}{c}\text { Alfa } \\
\text { de Cronbach }\end{array}$ & $\begin{array}{c}\text { Alfa de Cronbach basada } \\
\text { en elementos estandarizados }\end{array}$ \\
\hline Satisfacción situación laboral y vivienda & 0,766 & 0,784 \\
\hline Satisfacción de servicios básicos & 0,891 & 0,894 \\
\hline
\end{tabular}

Elaboración propia.

Se obtuvieron 195 respuestas de los habitantes de las 25 comunidades de Salinas de Guaranda, estos resultados se analizaron mediante estadística descriptiva a través del software SPSS versión 24.

Para el componente cualitativo, se realizó un muestreo teórico para la elección de los informantes, dado que se buscó que estos tuvieran como atributos fundamentales: haber conocido y recibido directamente transferencia de tecnología por parte de José Dubach y ser partícipes del desarrollo de la quesera principal de Salinas de la provincia de Bolívar desde sus inicios. 
El alcance de la investigación fue de tipo exploratorio-descriptiva, "dado que se realiza sobre un sistema que busca entender un fenómeno desconocido o novedoso" (Hernández-Sampieri, Fernández y Baptista 2014, 91). El aporte de la tecnología al desarrollo de la quesera principal en Salinas de Bolívar se centró en este fenómeno social. No obstante, al analizar estas influencias tecnológicas en la elaboración de quesos, tanto en el proceso inicial como posteriormente con la incorporación de las tecnologías del conocimiento, esta investigación también tiene un componente explicativo, pues indaga sobre los tipos de tecnologías y cómo su uso constituyó una variable en el desarrollo social de la población de Salinas de Bolívar.

El diseño de la investigación fue no experimental-longitudinal ya que no se manipularon las variables, sino que, por el contrario, solo se observaron y se estudiaron en su entorno natural para, posteriormente, poder analizarlas; estas observaciones no se redujeron a variables. El estudio recabó datos en diferentes puntos del tiempo sobre la quesera principal El Salinerito de Salinas de Bolívar para obtener, según su evolución, una deducción de sus causas y efectos en el aporte de la tecnología a su proceso de desarrollo. Asimismo, adoptó una perspectiva diacrónica, puesto que buscó estudiar este aporte a través del tiempo marcando tres hitos históricos importantes comprendidos en los períodos históricos: 1970-1980, 1980-1983, 1983-actualidad.

Por su parte, el diseño exploratorio-secuencial (DEXPLOS) "es comparativo, dado que en una primera fase se recolectan y analizan datos cualitativos para explorar el fenómeno" (Hernández-Sampieri, Fernández y Baptista 2014, 552). En esta etapa se recolectaron datos de la implementación de la tecnología de la quesera principal, generándose así una primera base de datos. En una segunda etapa, se recolectaron y analizaron los datos cuantitativos obtenidos del cuestionario aplicado, obteniendo una segunda base de datos. La interpretación final es el producto de la relación, integración y comparación de los resultados cualitativos y cuantitativos.

Se seleccionaron las siguientes herramientas para la recolección de datos mixtos. En una primera fase, se aplicaron entrevistas cualitativas semiestructuradas y abiertas realizadas a actores claves en el proceso salinero que han estado desde el inicio hasta la actualidad en la quesera principal, aquellos que pudieron recibir transferencia de tecnología por parte de José Dubach, y han participado a su vez en el desarrollo de la quesera principal de Salinas de 
Guaranda. Las preguntas se basaron en la indagación por experiencias, opiniones, valores, emociones, sentimientos, historias de vida y percepciones.

En una segunda fase, se aplicó una entrevista estructurada al gerente de la quesera principal, con el fin de entender cómo ha sido el desarrollo de la quesera con el aporte de las diferentes tecnologías. En una tercera fase, se aplicó un cuestionario (encuestas) que se dividió en tres partes: en la primera parte, se realizó una caracterización e identificación del entrevistado; en la segunda parte, se enumeraron tres preguntas que intentaron medir la percepción del impacto social y desarrollo de Salinas de Guaranda, y, en la tercera parte, se enumeraron cinco preguntas bajo la técnica del escalograma de Guttman con dos subcategorías (satisfacción de situación laboral y vivienda y satisfacción de servicios básicos), el cual se fundamentó en juicios ante los cuales los participantes debían externar su opinión seleccionando: muy satisfecho (4), satisfecho (3), poco satisfecho (2), nada satisfecho (1).

Adicionalmente, se aplicaron cinco preguntas dicotómicas de cuestionamiento directo sobre la implementación de la tecnología en la quesera principal en relación con la estabilidad laboral, vivienda, salud, educación y vías de acceso. Este conjunto de preguntas se aplicó a 195 pobladores de las 25 comunidades que cuentan con quesera en Salinas de Guaranda, incluyendo los trabajadores en la quesera principal de Salinas, El Salinerito.

En el apartado cualitativo se interpretaron datos a través de la identificación y codificación (Silver y Lewins 2007) de las once entrevistas desarrolladas y el material secundario consultado, arrojando conceptos y procesos que ayudaron a ampliar las teorías sobre la transferencia de tecnología por José Dubach en la implementación de la tecnología de la quesera principal El Salinerito y su contribución con el desarrollo de Salinas de Guaranda. La metodología incluyó procedimientos propios del método de la teoría fundamentada (Strauss y Corbin 2008) como un procedimiento general de análisis microscópico de los datos, codificación, definición de códigos y establecimiento de relaciones. Adicionalmente, se desarrolló una revisión de datos secundarios que implicó la revisión de documentos, registros públicos e históricos y archivos físicos sobre la trasferencia de tecnología en la elaboración de quesos, tipos de tecnologías utilizadas y demás aportes al desarrollo salinero. 


\section{Resultados y discusión}

\section{Transferencia de tecnologías por parte de José Dubach para el desarrollo de la quesera El Salinerito}

Hasta finales de los años 70 la parroquia Salinas de Guaranda se enfrentó con una tecnología deficiente, gracias a la cual el queso elaborado era de baja calidad y una acelerada caducidad, por lo que su comercialización no era un buen negocio. Todo ello ocurrió en una época en la que los precios eran ínfimos para el queso, factor que desencadenó una contracción de la actividad ganadera y la producción de leche de la zona, obligando a la migración hacia tierras más fértiles en las estribaciones de la cordillera Occidental (Soria e Illingwoth 1989). Dado que, en los meses de diciembre a mayo, durante los cuales predomina la época de lluvias, la actividad económica de obtener leña y maderas finas era inviable, los moradores se vieron obligados a retornar a la meseta andina, donde el pasto es abundante para el ganado y realizar pastoreo de doble propósito; este proceso se prolongó durante años, desperdiciando el potencial lechero de la zona por las dificultades a la hora de comercializar la leche y sus subproductos.

En 1972, la operación Mato Grosso llegó a una comunidad en la que las condiciones de vida de los habitantes de las zonas rurales de Salinas eran precarias. De cada dos niños, uno moría antes de cumplir el año de edad. No existía servicios de luz, agua potable, dispensario médico, centro de acopio, canales de riego, carreteras o trabajo gubernamental (Farzam Arbab 1989). Esta situación es confirmada por Antonio Polo, precursor de todo el desarrollo salinero y líder espiritual, quien arribó a Ecuador con esta operación: "Es difícil imaginar la situación que había en esa época, moría más que el cuarenta y cinco por ciento de los niños antes de los cinco años, entonces un dato que indica seguramente muchas cosas más" (Antonio Polo 2017, entrevista personal).

Cuando José Dubach inició sus labores en Salinas se encontró con un proceso de elaboración del queso deficiente, pero con una técnica tradicional ya establecida en los moradores salineros para la elaboración del quesillo, soportada en su conocimiento cotidiano o etnoconocimiento (Hess 1995). Este conocimiento se había adquirido en el ejercicio de sus actividades la- 
borales en la hacienda, adaptado a los recursos disponibles de las familias campesinas, las cuales producían cuando tenían tiempo o más leche de la que habitualmente se consumía en el hogar. La elaboración del quesillo se consideraba una actividad artesanal bajo un esquema de producción doméstica, con mano de obra familiar. Sin embargo, esta actividad de elaboración se convirtió en una herramienta indispensable para asegurar un recurso económico que permitiera la satisfacción mínima de las necesidades locales de cada familia salinera.

Gracias a la experticia obtenida en la implementación de otras queseras rurales, Dubach logró que su conocimiento fuera convertido en un objeto que permitiera utilizarse para la elaboración de un proceso que conllevó a la interacción con los aspectos cognitivos y de aprendizaje (Albino, Garavelli y Gorgoglione 2004) de los campesinos para la creación del proyecto de queserías rurales en Ecuador. Así, se inició una trasferencia de conocimiento gracias al movimiento y difusión de la tecnología (habilidades del hacer) para la elaboración de diferentes tipos de quesos, desde su iniciativa original a un contexto económico y social diferente (Becerra 2004).

El proceso de transferencia de conocimientos aportado por Dubach incluyó otros, como el tácito y explícito (Bueno, Plaz y Albert 2007), donde el conocimiento tácito en la elaboración de quesos se logra trasmitir mediante un proceso de socialización e interacción con las personas de la comunidad.

Este proceso de transferencia de conocimiento científico y tecnológico, tácito y explícito, constituyó la base fundamental para la trasferencia de tecnologías, innovación y habilidades de la comunidad Salinera, para la construcción de la quesera principal con la finalidad de mitigar los impactos económicos y sociales propios de la comunidad, como se representa en la figura 1.

La transferencia de tecnologías consensuadas bajo el acuerdo binacional entre Ecuador y Suiza logró trasladar al campesino de Salinas de Guaranda el capital intelectual y el saber-cómo (know-how), generando un nuevo conocimiento y aplicándolo a diferentes productos; esto dio como resultado procesos tecnológicos y formas sociales de producción para la instauración de los pilares en la quesera principal (Davenport y Prusak 1998). En esta transferencia de tecnología, José Dubach fue el proveedor y la comunidad de Salinas de Guaranda el receptor, la modalidad fue directa y siempre estuvo 
Figura 1

\section{Transferencia de conocimiento orientada al desarrollo social}

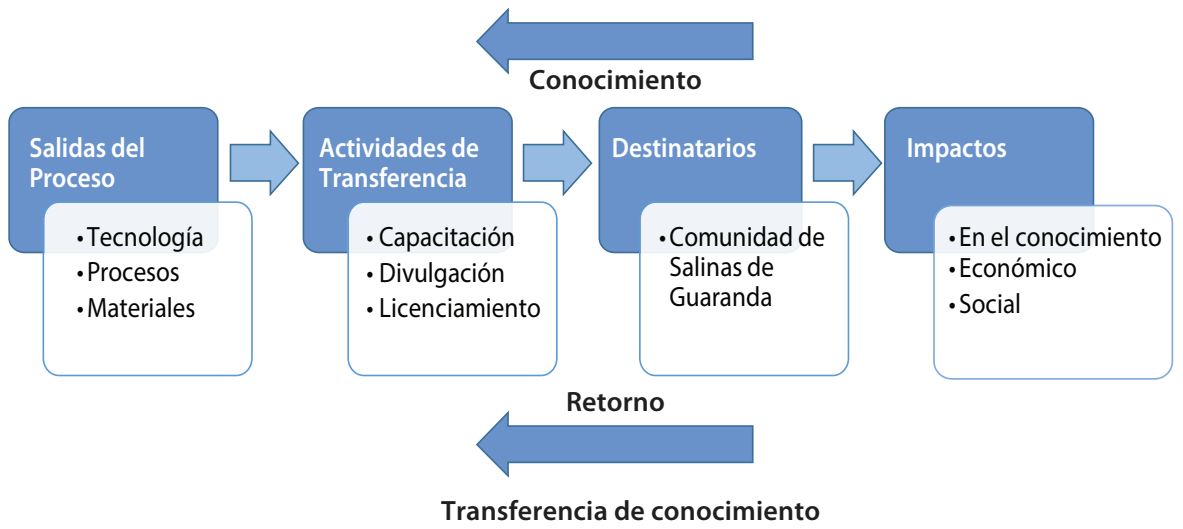

Elaboración propia.

influenciada por las motivaciones de tipo social y económico características de la población (Tapias-García 2016).

El proceso de capacitación desarrollado en Salinas de Guaranda fue, sobre todo, un proceso práctico, acorde con la lógica de prueba y error por medio de la que el campesino aplica y perfecciona su saber. Se fomentó la adquisición de conocimientos tecnológicos para la obtención de la destreza necesaria para desarrollar ese esquema pedagógico y, en consecuencia, optimizar la implementación del esquema productivo. Posteriormente, José Dubach decidió abrir la tienda Queseras de Bolívar en la ciudad de Quito para la venta de los quesos una vez a la semana, este fue un aporte importante para el proceso salinero.

La característica más importante de la transferencia de tecnologías por Dubach fue "la adecuación a las características sociales de aquellos que la utilizan y al recurso al cual es aplicada" (Cerdua 1980, 84). Esta trasferencia de tecnología se puede considerar plena, dado que se adaptó al sistema productivo de Salinas de Guaranda, y vertical, puesto que el conocimiento de fabricación de diversidad de quesos se utilizó para la creación de la primera quesera rural el Salinerito (Tapías-García 2016). Esta quesera, con base en 
las experiencias técnico-administrativas y de aplicación de los procedimientos probados, adaptó las herramientas desarrolladas y transferidas por José Dubach (inventor), representando y materializándose en un saber-como específico acorde con las condiciones de Salinas de Guaranda (Jarvie 2004). De este modo, se desarrollaron habilidades para la fabricación, culminado la elaboración diversificada de quesos en El Salinerito, enmarcando y resaltando una tecnología propia (Vieira Pinto 2008).

Las tecnologías del conocimiento transferidas por Dubach facilitaron el desarrollo, la implementación y, posteriormente, la utilización de tecnologías mecánicas y tecnologías humanas (Perrow 1967). Las tecnologías mecánicas destacaron la creación y utilización de máquinas, herramientas y equipos en las diferentes etapas del proceso productivo en la elaboración de los quesos como pasteurización, enfriamiento, maduración, batido, entre otros (Roberts y Grabowsky 1996).

Las tecnologías humanas trasferidas a los campesinos de la provincia de Bolívar se caracterizaron por la generación de las habilidades en ellos y la energía física usada en la producción de quesos (Roberts y Grabowsky 1996). Estas habilidades dotaron a los campesinos de posibles estrategias para fomentar el proceso con estándares de calidad, como es el caso de la temperatura adecuada en cada etapa productiva para influenciar el crecimiento de las bacterias, un factor indispensable en la fabricación de quesos, de cuyo manejo adecuado pueden depender el éxito o fracaso del producto final.

Parte de este análisis se desprende del trabajo desarrollado con el software Atlas.ti, con base en la revisión de documentos primarios, secundarios y las entrevistas a la población implicada. Para ello, se realizó una codificación abierta a través de los siguientes códigos: "José Dubach", "Maestro", "Calidad de vida", "Técnica", "Padre Antonio Polo", "Transferencia de tecnología", "Desarrollo de Salinas", "Tecnología", "Tecnología máquinas", "Tecnología innovación" y "Tecnología conocimiento". El código "José Dubach" tuvo la mayor recurrencia de citas seguido de "Calidad de vida" y "Tecnología".

Del código "José Dubach" se desglosa la red creada en la figura 2, dado que él trae consigo la técnica de elaboración de quesos permitiendo que se genere esta trasferencia de tecnología, lo cual es un factor directo de incidencia en el desarrollo de Salinas, territorio en el cual la calidad de vida marginal fue la principal motivación para desarrollar el proceso salinero. 
El padre Antonio Polo representa una figura asociada con la transferencia de tecnología y, al igual que José Dubach, una causa del "Desarrollo de Salinas". De la trasferencia de tecnología se desprenden las tecnologías mecánicas, tecnologías humanas (innovación), y las tecnologías del conocimiento. Cada una de estas tecnologías es interdependiente, coexisten e interrelacionan con el sistema de producción creado en la quesera principal El Salinerito, ejercen un impacto positivo en la organización de Salinas y en las prácticas productivas de los campesinos de toda la comunidad (Roberts y Grabowsky 1996).

Figura 2

Red transferencia de tecnologías José Dubach obtenida con Atlas.ti

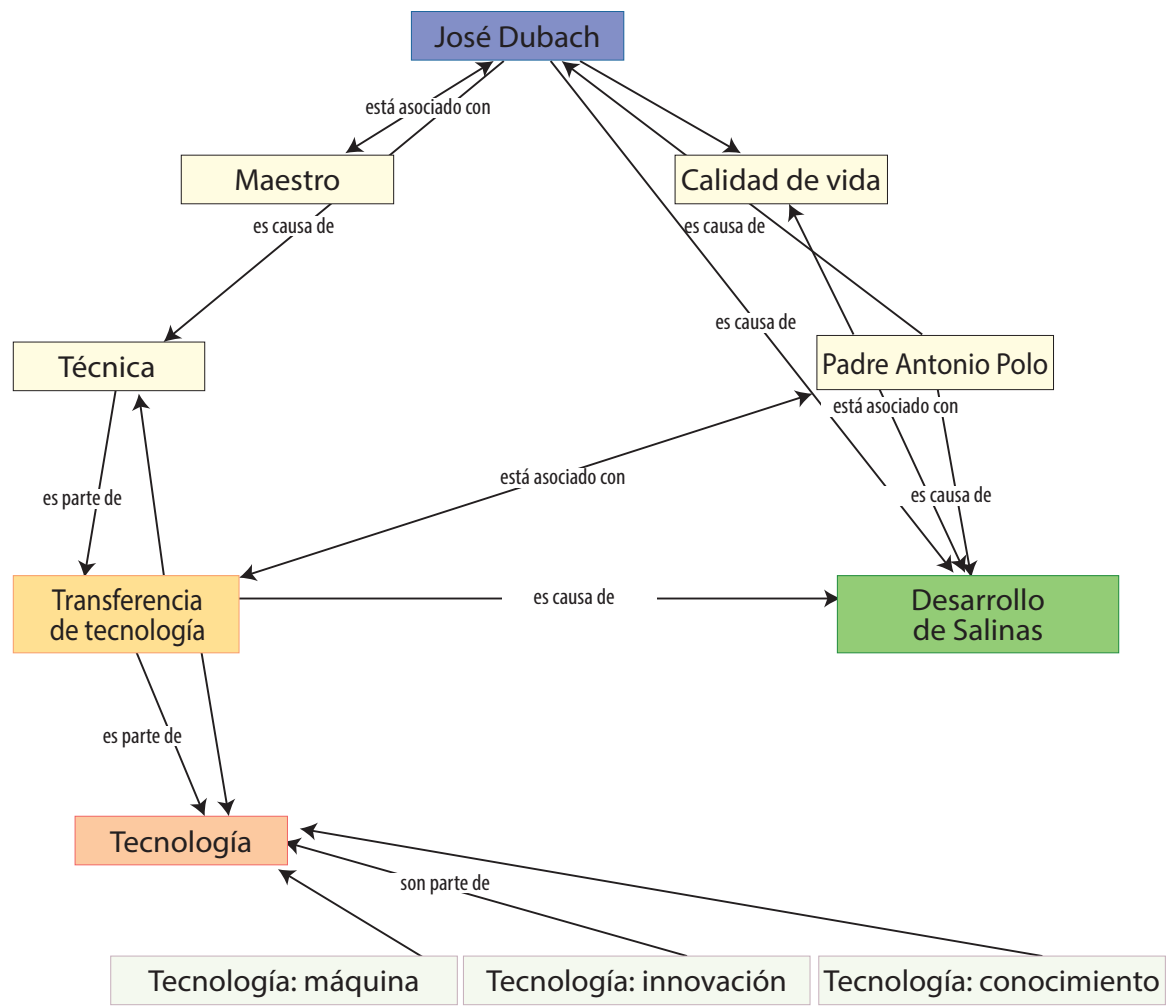

Elaboración propia. 


\section{Tipos de tecnologías utilizadas en las etapas del proceso comunitario de Salinas de la provincia de Bolívar}

El surgimiento y desarrollo de la quesera principal El Salinerito marcó un precedente en los miembros de la parroquia de Salinas, permitiendo que se formen conjuntamente como organización para mitigar la pobreza de la zona y actuando de manera comprometida en el inicio de la producción de quesos para lograr la trasformación de su entorno según sus necesidades de mejoramiento de la calidad de vida (Montero 2003). Se logró que las utilidades fueran reinvertidas en la quesera y en proyectos de educación, salud y mejoramiento de la infraestructura del pueblo (Naranjo 2012). Además, este proceso impulsó la creación descentralizada de la producción de quesos con la implementación de las tecnologías del conocimiento en las comunidades con el fin de implementar la construcción de queseras en cada una de ellas y adaptar el proceso productivo igual al que se realiza en la quesera principal, ayudadas de las tecnologías mecánicas y tecnologías humanas. A su vez, se produjo el inicio de otros procesos productivos como la fabricación de embutidos y confites, deshidratación de hongos y frutas, además de la promoción del turismo comunitario. Sin embargo, las organizaciones de Salinas de Guaranda "se desarrollaron como un medio para la satisfacción de sus necesidades y no como un fin que conduzca a una acumulación de capital en forma individual o de determinados grupos de poder" (Naranjo 2012, 110).

A través de la transferencia de tecnología, José Dubach impartió las tecnologías del conocimiento, tecnologías mecánicas y tecnologías humanas. Estas son implementadas en cada parte del proceso comunitario de Salinas. En este proceso comunitario se pueden encontrar hitos históricos importantes como se describen a continuación.

El primer hito abarca la llegada de la OMG, la creación de la primera cooperativa de ahorro y crédito, y el primer convenio entre Suiza y Ecuador (27 de febrero 1978-26 de febrero 1980) en el que se instalaron varias queserías, consolidando a El Salinerito como la principal, se desarrolló toda la cadena de valor, se capacitó a queseros y técnicos, además de la organización de los beneficiarios y el montaje de la primera tienda dedicada a la comercialización de sus productos. Este proceso productivo permitió que en 1982 se constituyera la Unión de Organizaciones de Salinas (UNORSAL), organiza- 
ción cooperativista de segundo grado que agrupaba en ese momento 22 organizaciones campesinas para el fortalecimiento de la actividad productiva. Estas cooperativas posibilitaron que las comunidades se incorporaran en todo el sistema de producción y fueran dueñas de sus organizaciones, participando en las decisiones sobre la conducción de su organización, viciándose, a su vez, de los resultados de la producción (Naranjo 2012).

El segundo hito abarca el segundo convenio Ecuador-Suiza (15 de marzo 1980-14 de marzo 1983) en el que se instalaron nuevas queserías en otras comunidades, dando continuidad al proceso con la capacitación del personal y ampliando el espacio de comercialización y fortalecimiento de relaciones interinstitucionales.

El tercer hito abarca el tercer y cuarto convenio Ecuador-Suiza (15 de marzo de 1983-31 de diciembre 1985) y (enero 1986-diciembre 1988), período durante el cual, con la asistencia técnica necesaria, se entregó el proyecto de queserías rurales a las organizaciones campesinas y se aseguró la continuación del apoyo de las instituciones públicas y privadas involucradas (Farzam Arbab 1989). Con este hito se logró el fortalecimiento del proceso comunitario de Salinas, del cual participan diversas unidades productivas que se lograron consolidar gracias a la primera unidad productiva en Salinas: la producción de una diversidad de quesos en El Salinerito a través de las tecnologías trasferidas por José Dubach.

\section{Impacto social en las comunidades de Salinas de la provincia de Bolívar con la implementación tecnológica en la quesera principal}

El cuestionario aplicado a un total de 195 personas de las 25 comunidades entre noviembre de 2017 y enero de 2018 incluyó, en una primera fase, preguntas dicotómicas, diseñadas con la finalidad de medir factores de estabilidad laboral, impacto positivo y reducción de la pobreza, influenciados por la quesera principal El Salinerito a partir del proceso de implementación tecnológica. 


\section{Factores laborales}

La actividad económica, contextualizada como una variable de la población de la comunidad de Salinas en esta investigación, indicó que es estable debido a la fabricación de quesos en El Salinerito. Esta actividad económica está representada en un $41,5 \%$ por pastoreo de ganado para producción de leche con doble propósito y un $36,9 \%$ en fabricación de diversos quesos y productos lácteos, lo que indica que el $78,4 \%$ de la actividad económica está relacionada con El Salinerito.

\section{Impacto social positivo en Salinas de Guaranda con el desarrollo de EI Salinerito}

A partir de los datos obtenidos se evidencia un importante impacto social en la comunidad, ya que se refleja un cambio sostenible a través de los 40 años de creación de El Salinerito, gracias al conjunto de intervenciones que desarrolló la unidad productiva de fabricación de diversos quesos (Tuan 2008). El impacto en Salinas de Guaranda es positivo como consecuencia del desarrollo de El Salinerito; el 99,5\% de las encuestas muestran un resultado positivo.

El impacto social en esta región está directamente relacionado con el desarrollo tecnológico (que incluye las tecnologías del conocimiento, mecánicas y humanas) de la quesera El Salinerito, siendo este un factor clave para su evolución y crecimiento económico. La actividad económica es estable, dado que el pastoreo de ganado y fabricación de quesos están inmersos en la cadena de valor de la unidad productiva y logran beneficiar a casi el $90 \%$ de la población salinera.

El mejoramiento de las condiciones de la población de Salinas se evidencia en la reducción de la pobreza actual, en comparación con los niveles de pobreza que manifestaban sus habitantes en 1978, cuando inició la primera planta de producción de quesos El Salinerito. El impacto ha sido positivo y se ha mantenido por 40 años, desatando el surgimiento de nuevos proyectos de desarrollo como la segunda planta de quesos del Salinerito en 2006, ubicada en el centro del pueblo, cerca del río Tigua, y la tercera planta en 2012. 


\section{Reducción de la pobreza con la implementación de la tecnología en El Salinerito}

La pobreza exhibe una clara reducción como consecuencia de la implementación tecnológica en El Salinerito, según la percepción del 88,72\% de los encuestados. Según el INEC $(2017,1)$, "En Ecuador una persona es considerada pobre por ingresos si percibe un ingreso familiar per capital menor de 84,49 USD mensuales", comparando los datos donde el $87,18 \%$ de las personas presentan ingresos en el rango de USD 386 a USD 772 y con la comparación de los indicadores del INEC, se afirma que la comunidad salinera no presenta pobreza por ingresos, gracias a la estabilidad de la actividad económica de sus habitantes producto de la implementación tecnológica de la quesera El Salinerito, pasando de una infraestructura inicial de 350 litros procesados a la producción actual de 10000 litros.

\section{Percepción de satisfacción de condiciones laborales}

El 87,18\% de los encuestados manifestó que está satisfecho con sus condiciones laborales actuales, dato ratificado por el 99,5\% que considera que tiene una actividad estable como consecuencia de la actividad productiva de El Salinerito y atribuye que esta condición se debe a la implementación tecnológica con una percepción del 94,36\% de aceptación.

- Vivienda: el 73,85\% de los encuestados manifestó que está satisfecho con sus condiciones de vivienda, puesto que el $96,92 \%$ vive en una casa, con un $91,28 \%$ que habita en una casa propia. E1 77,95\% de la población consideró que las condiciones de vivienda actuales están relacionadas con la implementación tecnológica en El Salinerito.

- Salud: el 75,90\% de los encuestados manifestó poca satisfacción con las condiciones de acceso a salud, y se establece que no influye la implementación de la tecnología del Salinerito con el acceso a los servicios de salud con un $80,51 \%$.

- Educación: el 81,03\% de los encuestados estaba poco satisfecho con las condiciones de acceso a servicios de educación y se establece que no influye la implementación de la tecnología de El Salinerito con el acceso a los servicios de salud con un $83,08 \%$. 
- Acceso vial: el 77,95\% de los encuestados estaba poco satisfecho con las condiciones de servicios de acceso vial, pero sí influye la implementación de la tecnología de El Salinerito con el acceso a este servicio con un $54,36 \%$.

La satisfacción con las condiciones laborales y de vivienda actual son altas, el rango resultante de esta investigación cambió de satisfecho a poco satisfecho. Por tanto, se evidenció que los encuestados se inclinaron hacia valores elevados en la medida de la satisfacción de estas condiciones. Además, la media de los participantes para las condiciones laborales fue de 2,87 mientras que para la vivienda actual fue de 2,74 y la mediana que representa el valor de la variable de posición central en un conjunto de datos, en este caso es de valor 3, lo cual confirma la tendencia de la muestra hacia valores altos de escala. Se podría atribuir a que esta medida satisfactoria en las condiciones laborales y de vivienda son desencadenadas por la actividad productiva de pastoreo, fabricación de quesos y productos lácteos; actividades inmersas en la cadena de valor en la producción de quesos en El Salinerito. La implementación tecnológica en El Salinerito ha desencadenado un aumento en la capacidad de procesamiento de leche, generando una mejora significativa de las condiciones de la población de Salinas de Guaranda, que puede ser perdurable en el tiempo (Berner et al. 2004), como se evidencia en los 40 años desde la constitución de la quesera principal.

Las condiciones de salud, educación y acceso vial tuvieron poca satisfacción; el rango de esta investigación se modificó de satisfecho a nada satisfecho. Los individuos se inclinaron hacia valores bajos, donde la media de los participantes fue para salud 2,17; educación, 2,14 y acceso vial, 2,19 con una mediana de 2 , lo cual confirma la tendencia de la muestra hacia valores bajos de escala.

La baja respuesta acerca de las condiciones de salud, educación y vías se atribuye a que, si bien la implementación tecnológica en El Salinerito desprendió el proceso comunitario y el desarrollo social en Salinas de Guaranda, al aprovechar las capacidades, recursos y oportunidades de los moradores y generar nuevos conocimientos en el proceso de elaboración de quesos para permitir el acceso a recursos que faciliten un nivel de vida aceptable y salir de la pobreza absoluta en la que estaban inmersos (Escobar 2014), no es sobre la quesera principal que recae la obligación del mejoramiento de las 
condiciones de vida en estos ámbitos, sino sobre el Estado, como promotor y coordinador (Ochoa 2006). Sin embargo, para lograr esta promoción de bienestar en Salinas de Guaranda, el desarrollo social se estructuró a partir de los propios actores sociales locales y no solo por la planificación centralizada del Estado (Tenorio 2016).

El impacto social en las comunidades de Salinas de la provincia de Bolívar con la implementación tecnológica en la quesera principal, en general se califica como positiva, desencadenada a su vez por un desarrollo local con la articulación, coordinación e inserción de los emprendimientos comunitarios de las diferentes asociaciones y cooperativas creadas para tal fin del grupo Salinas, con una integración socioeconómica que permitió la reconstrucción del tejido social al superar la pobreza extrema por ingreso hasta las condiciones actuales de desarrollo.

El instrumento para la medición del impacto de la implementación tecnológica se validó mediante herramientas estadísticas como el Alfa de Conbrach, obteniendo resultados superiores a 0,7 lo que muestra la fiabilidad de este método. Adicionalmente, se evidencia los coeficientes de Pearson como se muestra en la figura 3.

Los resultados obtenidos a través de la encuesta referida a la evaluación de las condiciones laborales, vivienda, salud, educación y acceso vial, son los siguientes:

- La existencia de una actividad económica estable genera un impacto social positivo, al igual que una percepción de reducción de la pobreza con la implementación tecnológica en la quesera principal El Salinerito.

- La satisfacción de condiciones laborales obtuvo una media de 2,87 y, según la escala de Guttman propuesta, es un valor de satisfacción. Se considera que la implementación tecnológica en de El Salinerito influye en esta satisfacción.

- La satisfacción de condiciones de vivienda obtuvo una media de 2,74 y, según la escala de Guttman propuesta, es un valor de satisfacción. Se considera que la implementación tecnológica de El Salinerito influye en esta satisfacción.

- La satisfacción de condiciones de acceso a servicios de salud obtuvo una media de 2,17 y, según la escala de Guttman propuesta, es un valor de 
Tabla 3

\section{Correlaciones}

\begin{tabular}{|c|c|c|c|c|}
\hline & & $\begin{array}{l}\text { ¿En qué medida } \\
\text { usted está } \\
\text { satisfecho con } \\
\text { las condiciones } \\
\text { de acceso a } \\
\text { los servicios de } \\
\text { salud actuales } \\
\text { en Salinas de } \\
\text { Guaranda? }\end{array}$ & $\begin{array}{l}\text { ¿En qué medida } \\
\text { usted está } \\
\text { satisfecho con } \\
\text { las condiciones } \\
\text { de acceso a } \\
\text { los servicios de } \\
\text { salud actuales } \\
\text { en Salinas de } \\
\text { Guaranda? }\end{array}$ & $\begin{array}{l}\text { ¿En qué medida } \\
\text { usted está } \\
\text { satisfecho con } \\
\text { las condiciones } \\
\text { de acceso } \\
\text { vial actuales } \\
\text { en Salinas de } \\
\text { Guaranda? }\end{array}$ \\
\hline $\begin{array}{l}\text { ¿En qué medida } \\
\text { usted está } \\
\text { satisfecho con } \\
\text { las condiciones } \\
\text { de acceso a } \\
\text { los servicios de } \\
\text { salud actuales } \\
\text { en Salinas de } \\
\text { Guaranda? }\end{array}$ & $\begin{array}{l}\text { Correlación de } \\
\text { Pearson } \\
\text { Sig. (bilateral) } \\
\text { N }\end{array}$ & 195 & $\begin{array}{r}0,739 \\
0,000 \\
195\end{array}$ & $\begin{array}{r}0,641 \\
0,000 \\
195\end{array}$ \\
\hline $\begin{array}{l}\text { ¿En qué medida } \\
\text { usted está } \\
\text { satisfecho con } \\
\text { las condiciones } \\
\text { de acceso a } \\
\text { los servicios de } \\
\text { salud actuales } \\
\text { en Salinas de } \\
\text { Guaranda? }\end{array}$ & $\begin{array}{l}\text { Correlación de } \\
\text { Pearson } \\
\text { Sig. (bilateral) } \\
\text { N }\end{array}$ & $\begin{array}{r}0,739 \\
0,00 \\
195\end{array}$ & 195 & $\begin{array}{r}0,835 \\
0,000 \\
195\end{array}$ \\
\hline $\begin{array}{l}\text { ¿En qué medida } \\
\text { usted está } \\
\text { satisfecho con } \\
\text { las condiciones } \\
\text { de acceso } \\
\text { vial actuales } \\
\text { en Salinas de } \\
\text { Guaranda? }\end{array}$ & $\begin{array}{l}\text { Correlación de } \\
\text { Pearson } \\
\text { Sig. (bilateral) } \\
\text { N }\end{array}$ & $\begin{array}{r}0,641 \\
0,000 \\
195\end{array}$ & $\begin{array}{r}0,835 \\
0,00 \\
195\end{array}$ & 195 \\
\hline
\end{tabular}

Elaboración propia. 
poca satisfacción. No se considera que la implementación tecnológica en El Salinerito haya influido en la satisfacción de acceso a este servicio.

- La satisfacción de condiciones de acceso a educación obtuvo una media de 2,14 y según la escala de Guttman propuesta es un valor de poca satisfacción. No se considera que la implementación tecnológica de El Salinerito influya sobre la satisfacción de acceso a este servicio.

- La satisfacción de condiciones de acceso vial obtuvo una media de 2,19 y, según la escala de Guttman propuesta, es un valor de poca satisfacción. Se considera que la implementación tecnológica de El Salinerito influye en la satisfacción de acceso vial.

- Se pudo establecer una correlación positiva considerable entre salud y educación con un coeficiente de Pearson de 0,739 , con un coeficiente significativo al nivel de 0,01 .

- Se pudo establecer una correlación positiva media entre salud y acceso vial con un coeficiente de Pearson de 0,641 , con un coeficiente significativo al nivel de 0,01 .

- Se pudo establecer una correlación positiva considerable entre educación y acceso vial con un coeficiente de Pearson de 0,835 , con un coeficiente significativo al nivel de 0,01 .

Los métodos empleados, que integran lo cualitativo y lo cuantitativo, permiten trazar el camino del desarrollo de la actividad productiva de Salinas de Guaranda, no solo mostrando los porcentajes de ganancias y desarrollo, sino, también, el mejoramiento de la calidad de vida de los campesinos salineros, quienes antes de entrar en este proceso de implementación tecnológica vivían sumidos en la pobreza y la incertidumbre financiera.

Finalmente, en la investigación se determinó la importancia de José Dubach en el proceso de desarrollo de Salinas de Guaranda al ser el precursor de la trasferencia de tecnología, base del exitoso modelo El Salinerito. Se deja abierta la posibilidad de un futuro estudio sobre la instauración de la marca comercial José Dubach en la presentación de quesos y su incorporación en el Grupo Salinas. 


\section{Conclusiones}

Gracias a la actividad de elaboración de quesos, Salinas de Guaranda logró desencadenar el proceso de desarrollo de la región. La implementación tecnológica se basó en la trasferencia de tecnologías mecánicas, humanas y del conocimiento, impartidas por José Dubach.

Las tecnologías mecánicas permitieron la consolidación y optimización de las distintas etapas del proceso productivo, acompañadas de técnicas de innovación compatibles con la cultura agrícola de los campesinos salineros. Por su parte, las tecnologías humanas, la organización interna y los valores culturales del grupo social campesino, fueron el sustento del exitoso modelo importado a Ecuador por el técnico suizo. Ambas vertientes tecnológicas se enmarcan en las tecnologías del conocimiento, en las que los significados y conceptos usados en la producción de quesos fueron la base fundamental, pues incorporaron el mejoramiento de los quesos tradicionales y demás procesos productivos: 1 . apertura a las acciones de capacitación; 2 . mejoramiento de pastos y ganado; 3 . mejoramiento de las condiciones y acceso al crédito; 4 . eliminación de intermediarios de comercialización; y, 5. mayor integración al campesino con las ciudades principales, para así conseguir un impacto significativo al pequeño productor, mejorando su situación socioeconómica.

El proceso comunitario se desarrolla por la actividad productiva de la quesera principal El Salinerito, hecho a partir del cual se genera la necesidad de agrupar a las queserías con el fin de ayudar a los campesinos interesados en seguir las tecnologías aplicadas en la construcción de la quesera principal, para que se copie el modelo y de este modo las familias no se desplacen a otros lugares, pues la instalación de una quesera en cada comunidad proporciona trabajo continuo, dotando así a los pobladores de una actividad económica estable que proporciona ingreso fijos mensuales a la familias, que garantizan la atención y satisfacción de sus necesidades básicas individuales y el sostenimiento de la actividad productiva comunitaria.

El aporte de las tecnologías en el desarrollo de la quesera principal mejoró los ingresos de los pequeños productores de leche, gestionó el proceso comunitario que promovió la puesta en marcha de otras actividades productivas, creó puestos de trabajo, formó hábitos de higiene y precisión, e intro- 
dujo una actividad social económica que permitió fortalecer la organización campesina para que se anulara la migración. Todo ello, se traduce en una clara disminución de los índices de pobreza en las comunidades implicadas, lo que supone una mejor calidad de vida para los salineros.

Los resultados obtenidos a través de la encuesta referida a la evaluación de las condiciones laborales, vivienda, salud, educación y acceso vial son los siguientes: El Salinerito ha influido en la satisfacción de acceso en una media de 2,19 y, según la escala de Guttman propuesta, es un valor de poca satisfacción. Se considera que la implementación tecnológica de El Salinerito influye en la satisfacción de acceso a estos servicios básicos.

\section{Referencias}

Albino, Vito, Achille Garavelli y Michele Gorgoglione. 2004. "Organization and Technology in Knowledge Transfer”. Benchmarking International Journal 11 (6): 584-600. https://doi. org/10.1108/14635770410566492.

Becerra, Martha. 2004. La trasferencia de tecnología en Japón: conceptos y enfoques. Monterrey: Universidad Autónoma de Nuevo León.

Berner, Heidi, Ryan Cooper, Marcela Guzmán y Nelson Guzmán. 2004. Metodología de evaluación de impacto. Santiago: Gobierno de Chile.

Bueno, Eduardo, Reinaldo Plaz Landaeta y José Albert. 2007. "Modelo de gobierno del conocimiento y su aplicación en las OTRIS: dos casos de implantación". Economía Industrial (366): 97-112. https://bit.ly/3wVEhgJ.

Celina Oviedo, Heidi, y Adalberto Campo Arias. 2005. "Aproximación al uso del coeficiente Alfa de Conbrach". Revista Colombiana de Psiquiatría 24 (4): 572-580. https://bit.ly/3ia4Vhz.

Cerdua, Juan Carlos. 1980. Tecnología apropiada para el desenvolvimiento comunitario. Río de Janeiro: ICCA.

Collins, Paul, Jerald Hage y Frank Hull. 1988. "A Framework for Analyzing Technical Systems in Complex Organizations". En Research in Sociology of Organizations, editado por Samuel Bacharach y Nancy DiTomaso, 81-100. Greenwich: JAI Press.

Davenport, Thomas, y Laurence Prusak. 1998. Working Knowledge: How Organizations Manage What They Know. Boston: Harvard Business School Press.

Departamento de Ingeniería Marítima de España. 2018. "Tecnología mecánica”. Accedido junio de 2018. https://bit.ly/34Ed0Dg.

EC Instituto Nacional de Estadística y Censos (INEC). 2017. Indicadores de pobreza y desigualdad en Ecuador. Quito: INEC. 


\section{Adriana Delgado}

EC Secretaría Nacional de Planificación y Desarrollo (SENPLADES). 2015. GAD para Salinas. Quito: SENPLADES.

Escobar, Arturo. 2014. La invención del desarrollo. Popayán: Universidad del Cauca.

Farzam Arbab. 1989. Celater: marco conceptual. Cali: CELATER.

GAD Salinas. 2015. “Actualización del plan de desarrollo y ordenamiento territorial de la parroquia rural Salinas”. Salinas: GAD parroquial rural Salinas.

González García, Marta Isabel, José Luis Luján y José Antonio López Cerezo. 1996. Una introducción al estudio social de la ciencia y tecnología. Madrid: Tecnos.

Hernández, Roberto, Carlos Fernández y María del Pilar Baptista. 2014. Metodología de la investigación. Ciudad de México: McGraw Hill.

Hess, David. 1995. Science and Technology in a Multicultural World. The Cultural Politics of Facts and Artifacts. Nueva York: Columbia University Press.

Libraryhouse. 2008. Metrics for the Evaluation of Knowledge Transfer Activities at Universities. Accedido noviembre de 2017. https://bit.ly/3pShvUA.

Montero, Maritza. 2003. Teoría y práctica de la psicología comunitaria: la tensión entre comunidad y sociedad. Buenos Aires: Editorial Paidós.

Naranjo, Klever Efraín. 2012. Cultura local y gestión. Quito: Universidad Andina Simón Bolívar, Sede Ecuador.

North, Lisa, y John Cameron. 2008. Desarrollo rural y neoliberalismo. Ecuador desde una perspectiva comparativa. Quito: Corporación Editora Nacional.

Ochoa, Sara María. 2006. Investigación sobre desarrollo social. Ciudad de México: Centro de Estudios Sociales y de Opinión Pública. https://bit.ly/3g0TJ4i.

Olarte, Luis Alberto. 1996. "Transferencia de tecnología”. Ingeniería e Investigación: 4: 3548. https://bit.ly/3wqYTO0.

Perrow, Charles. 1967. "A Framework for the Comparative Analysis of Organizations". American Sociological Review 32 (2): 194-208. https://doi.org/10.2307/2091811.

Polo, Antonio. 2002. La puerta abierta: 30 años de aventura misionera y social en Salinas de Bolivar-Ecuador. Quito: Abya-Yala.

Price, John de Solla. 1980. "Ciencia y tecnología: distribuciones e interrelaciones". En Estudios sobre sociología de la ciencia, coordinado por Barry Barnes, 163-177. Madrid: Alianza Editorial.

Roberts, Karlene, y Martha Grabowsky. 1996. "Organizations, Technology and Structuring”. En The Sage Handbook of Organization Studies, editado por Steward R. Clegg, Cynthia Hardy, Thomas B. Lawrence y Walter R. Nord, 314-333. Londres: Sage Publications.

Sagasti, Francisco. 2011. Ciencia, tecnología, innovación: politicas para América Latina. Ciudad de México: Fondo de Cultura Económica.

Silver, Christina, y Ann Lewins. 2007. Using Software in Qualitative Research. A Step-ByStep Guide. Thousand Oaks: Sage Publications Inc.

Soria, Román, y Vicente Illingworth. 1989. Queserías rurales en los Andes: la experiencia de Salinas-Ecuador. Cali: CELATER. 
Aporte tecnológico al desarrollo de la quesera principal de Salinas, en la provincia de Bolívar

Strauss, Anselm, y Juliet Corbin. 2008. Basics of Qualitative Research: Techniques and Procedures for Developing Grounded Theory. Thousand Oaks: Sage Publications.

Tapias-García, Heberto. 2016. "Transferencia de tecnología”. Revista Facultad de Ingeniería Universidad de Antioquia 12 (13): 9-15. https://bit.ly/3cdHgJs.

Teddlie, Charles, y Fen Yu. 2007. "Mixed Methods Sampling: A Typology with Examples”. Journal of Mixed Methods Research 1 (1): 77-100. https://doi.org/10.1177/1558689806292430.

Tenorio, Fernando Guilherme. 2016. Uma alternativa: gestão social. Río Grande del Sur: Editora Unijuí.

Tuan, Melinda T. 2008. Measuring and/or Estimating Social Value Creation: Insights into Eight Integrated Cost Approaches. Seattle: Bill \& Melinda Gates Foundation. https://gates. ly/3fI1A7P.

Vieira Pinto, Alvaro. 2008. O conceito de tecnologia. Río de Janeiro: Editora Contraponto.

Wenk, Edward. 1989. Tradeoffs: Imperative of Choice in a High-Tech World. Baltimore: Johns Hopkins University Press.

Yamane, Taro. 1967. Statistics: An Introductory Analysis. Nueva York: Harper \& Row.

\section{Entrevistas realizadas por la autora}

Antonio Polo, 6 de noviembre de 2017.

Jorge Nasabanda, 6 de noviembre de 2017.

Cesar Cumbay, 6 de noviembre de 2017.

Orlando Córdoba, 6 de noviembre de 2017.

Dolores Vargas, 6 de noviembre de 2017.

José Colombo, 6 de noviembre de 2017.

Armando Tualombo, 24 de noviembre de 2017. 